\title{
Data from 3D Computer Vision
}

\author{
Henrik Aanaes \\ Technical University of Denmark \\ Anker Engelunds Vej 1 Bygning 101A, 2800 Kgs. Lyngby, Denmark \\ aanes@dtu.dk
}

In this talk I will start out by briefly arguing the need for more empirical data in computer vision, especially in the domain of 3D estimation. Following this, I will present some of the work we have done at our lab with respects to addressing this problem. In particular I will focus on our work on evaluating salient feature matching and multiple view stereo. I will then proceed by arguing that we do not only need to address geometry, but also radiometry when capturing 3D objects, and present some of the work we have done in this respects. I will conclude with a pointers to what I see as open research questions, based on what I have presented. 